\title{
Association of common genetic variants related to atrial fibrillation and the risk of ventricular fibrillation in the setting of first ST-elevation myocardial infarction
}

Reza Jabbari ${ }^{1 *}$ (D, Javad Jabbari ${ }^{1}$, Charlotte Glinge ${ }^{1}$, Bjarke Risgaard ${ }^{1}$, Stefan Sattler ${ }^{1}$, Bo Gregers Winkel ${ }^{1}$, Christian Juhl Terkelsen ${ }^{2}$, Hans-Henrik Tilsted ${ }^{3}$, Lisette Okkels Jensen ${ }^{4}$, Mikkel Hougaard ${ }^{4}$, Stig Hauns $\varnothing^{1,5}$, Thomas Engstrøm ${ }^{1,7}$, Christine M. Albert ${ }^{6}$ and Jacob Tfelt-Hansen ${ }^{1}$

\begin{abstract}
Background: Cohort studies have revealed an increased risk for ventricular fibrillation (VF) and sudden cardiac death (SCD) in patients with atrial fibrillation (AF). In this study, we hypothesized that single nucleotide polymorphisms (SNP) previously associated with AF may be associated with the risk of VF caused by first ST-segment elevation myocardial infarction (STEMI).

Methods: We investigated association of 24 AF-associated SNPs with VF in the prospectively assembled case-control study among first STEMI-patients of Danish ancestry.

Results: We included 257 cases (STEMI with VF) and 537 controls (STEMI without VF). The median age at index infarction was 60 years for the cases and 61 years for the controls $(p=0.100)$. Compared to the control group, the case group was more likely to be male ( $86 \%$ vs. $75 \%, p=0.001)$, have a history of AF ( $7 \%$ vs. $2 \%, p=0.006)$ or hypercholesterolemia (39\% vs. $31 \%, p=0.023)$, and a family history of sudden death ( $40 \%$ vs. $25 \%, p<0.001)$. All 24 selected SNPs have previously been associated with AF. None of the 24 SNPs were associated with the risk of VF after adjustment for age and sex under additive genetic model of inheritance in the logistic regression model.

Conclusion: In this study, we found that the 24 AF-associated SNPs may not be involved in increasing the risk of VF. Larger VF cohorts and use of new next generation sequencing and epigenetic may in future identify additional AF and VF risk loci and improve our understanding of genetic pathways behind the two arrhythmias.
\end{abstract}

Keywords: Ventricular fibrillation, Atrial fibrillation, Myocardial infarction, ST-elevation myocardial infarction, Sudden cardiac death, Genetics, Single nucleotide polymorphisms

\section{Background}

Cohort studies have revealed an increased risk for ventricular fibrillation (VF) [1-4] and sudden cardiac death (SCD) [5-7] in patients with atrial fibrillation (AF). In the Danish GEVAMI (GEnetic causes of Ventricular Arrhythmias in patients with first ST-elevation Myocardial Infarction) study we have previously demonstrated a strong association between AF and VF before

\footnotetext{
* Correspondence: rezajabbari77@gmail.com

${ }^{1}$ Department of Cardiology, Rigshospitalet, Copenhagen University Hospital, Blegdamsvej 9, 2100 Copenhagen $\varnothing$, Denmark

Full list of author information is available at the end of the article
}

primary percutaneous coronary intervention (PPCI) with an odds ratio (OR) of 2.8 (95\% CI: 1.10-7.30) [1]. The GEVAMI study population includes patients presenting with first ST-segment elevation myocardial infarction (STEMI) that do (case) or do not (control) develop VF prior to primary angioplasty.

Heritable factors and cardiac channelopathies have been suggested as cause of VF and AF [8, 9]. Genome-wide association studies (GWAS) in individuals of European, $[10,11]$ Asian, [12] and African American [13] descent have identified several genomic regions with $\mathrm{AF}$. Especially single nucleotide polymorphisms (SNP) on 
chromosomes 4q25 (PITX2) have strong association with AF $[9,14]$, but also with higher risk of SCD [15]. Furthermore, SCN5A and SCN10A which encode the transient sodium channel $\left(\mathrm{I}_{\mathrm{Na}}\right)$ and play a pivotal role in membrane depolarization during cardiac action potentials have also been associated with AF, [16, 17] VF [17], and also Brugada Syndrome $[18,19]$.

In this study, we hypothesized SNPs that previously were associated with AF can affect the risk of VF in the GEVAMI study population and thereby suggest a shared genetic pathway.

\section{Methods}

\section{Study population}

The study population and design has been described previously $[1,20]$. In brief, the Danish GEVAMI study is an ongoing nationwide prospectively collected casecontrol study among patients with first STEMI between the ages of 18 and 80 years $[1,20]$. The case group are STEMI patients who had VF or sustained ventricular tachycardia (VT; $n=6)$ with cardiac arrest within the first $12 \mathrm{~h}$ of symptoms of STEMI before PPCI, and the control group are STEMI patients who did not have VF/ VT. All the patients are collected at all four PPCI centers in Denmark. Both groups were required to have cardiac symptoms lasting $\leq 12 \mathrm{~h}$, acute STEMI on ECG, which required acute PPCI. Baseline demographics and previous medical history are collected by research coordinators utilizing pre-designed questionnaires and whole blood is collected for genetic analysis.

\section{DNA extraction and SNP genotyping}

The methods for DNA extraction and also the SNP genotyping and analysis of this study have been described previously in detail [20]. In brief, we used whole blood and isolated the total genomic DNA using LGC's Kleargene $^{\text {Tm }}$ silica-based DNA extraction technique, which was performed at LGC Genomics. Isolated DNA was then analyzed using UV spectrophotometry to estimate both the quality and quantity of the DNA and normalized.

Based upon the results of previous genetic association studies, 24 common genetic variants known to be associate with AF $[9-12,14,16,17,21-27]$ were selected for SNP genotyping in both the cases and controls. SNP genotyping was performed using $\mathrm{KASP}^{\mathrm{m} \mathrm{m}}$ genotyping assays from LGC genomics (http://www.lgcgenomics.com). For each SNP two allele specific forward primers and one reverse primer were designed (LGC Genomics, Hoddesdon, UK) as described in detail previously [20]. All assays were conducted without any knowledge of case or control status. Genotypes for all SNPs passed our quality-control threshold (call-rate $\geq 94 \%$; Hardy-Weinberg equilibrium $P>0.05$ in control subjects).

\section{Statistical analysis}

Medians or proportions of baseline and presenting characteristics were computed for cases and controls, and significance of associations were tested using the Wilcoxon rank-sum test for continuous variables and the $\mathrm{x}^{2}$ test or Fisher exact test (where appropriate) for categorical variables. A two-tailed $p$ value $\leq 0.05$ was considered statistically significant. A logistic regression model were constructed to estimate OR for the association between each SNP and VF using an additive model (per copy allele frequency) of inheritance, as described previously [20]. The logistic regression model was adjusted for age and sex. All analyses were performed using the Stata software package version 12.0 (StataCorp).

\section{Results}

\section{Clinical characteristics of the cohort}

The clinical characteristics of the cohort are described previously $[1,20]$. In total, 257 cases (35 women and 222 men) with VF caused by first STEMI and 537 STEMI controls (131 women and 426 men) who did not developed VF were included in this prospective assembled study. Table 1 shows the baseline characteristics of the GEVAMI cohort. The median age of the cases was 60 (interquartile range (IQR); 53-68) years versus 61 (IQR; 52-66) years for the controls. As reported previously, compared to the controls, cases were more likely to be male, have atrial fibrillation or hypercholesterolemia, and a family history of sudden death [1]. The statin therapy was higher in the cases compared to the controls, most likely due to the higher degree of hypercholesterolemia in the cases. Levels of average weekly alcohol intake were higher in the cases (6 units/week) compared to the controls (3 units/week) $(P=0.001)$. Regarding other cardiovascular risk factors such as smoking, diabetes, and hypertension, did cases not differ significantly from the controls.

\section{Common genetic variants associated with VF}

Comprehensive panels of 24 common variants were genotyped in 794 individuals in the GEVAMI population with Danish descent (Table 2). All the selected SNPs were reported to be associated with AF. The overall call rate was $\geq 95 \%$. Table 2 shows the risk allele frequencies and the OR for each SNP. The rs6795970 SNP risk allele A did not exist in our cohort although the average callrate was $97.8 \%$. None of the 24 common genetic variants were associated with the risk of VF in the GEVAMI population after multivariable adjustment (Table 2).

\section{Discussion}

In this study, we investigated the role of 24 common SNPs previously associated with $\mathrm{AF}$ and their associations with 
Table 1 Baseline characteristics of the cohort

\begin{tabular}{|c|c|c|c|}
\hline Variables & Cases $(n=257)$ & Controls $(n=537)$ & $P$ value \\
\hline Female sex, No. (\%) & $35(14)$ & $131(25)$ & 0.001 \\
\hline Median Age at index infarction, y (IQR) & $60(53-68)$ & $61(52-66)$ & 0.100 \\
\hline \multicolumn{4}{|l|}{ Cardiovascular risk profile } \\
\hline Body mass index $\left(\mathrm{kg} / \mathrm{m}^{2}\right),(\mathrm{QQR})$ & $27.2(25-29)$ & $26.7(24-29)$ & 0.400 \\
\hline Smoking (pack year), (IQR) & $25(5-41)$ & $25(6-42)$ & 0.200 \\
\hline \multicolumn{4}{|l|}{ Smoking, No. (\%) } \\
\hline Never & $38(16)$ & $108(20)$ & \multirow[t]{3}{*}{0.300} \\
\hline Past & $69(28)$ & $133(25)$ & \\
\hline Current & $136(56)$ & $290(55)$ & \\
\hline Alcohol per week, (unit*, IQR) & $6(1-15)$ & $3(0-9)$ & $<0.001$ \\
\hline \multicolumn{4}{|c|}{ Alcohol units per week (categorized), No. (\%) } \\
\hline Non-drinkers & $46(19)$ & $143(27)$ & \multirow[t]{4}{*}{$<0.001$} \\
\hline Normal (1-7) & $90(38)$ & $242(46)$ & \\
\hline Moderate High (8-14) & $41(17)$ & $70(13)$ & \\
\hline High $(>15)$ & $60(26)$ & $73(14)$ & \\
\hline Diabetes, No. (\%) & $30(12)$ & $47(9)$ & 0.200 \\
\hline Hypertension, No. (\%) & $102(41)$ & $184(35)$ & 0.070 \\
\hline COPD, No. (\%) & $12(5)$ & $30(6)$ & 0.700 \\
\hline Hypercholesterolemia, №. (\%) & $97(39)$ & $165(31)$ & 0.023 \\
\hline Stroke, No. (\%) & $18(7)$ & $26(5)$ & 0.200 \\
\hline Atrial fibrillation, No. (\%) & $16(7)$ & $10(2)$ & 0.006 \\
\hline Depression, No. (\%) & $28(11)$ & $65(12)$ & 0.700 \\
\hline Epilepsy, №. (\%) & $4(2)$ & $5(1)$ & 0.500 \\
\hline \multicolumn{4}{|l|}{ Family History, No. (\%) } \\
\hline Sudden death & $94(40 \%)$ & $128(25 \%)$ & $<0.001$ \\
\hline Myocardial infarction & $90(40 \%)$ & $195(38 \%)$ & 0.600 \\
\hline Stroke & $36(16 \%)$ & $75(15 \%)$ & 0.600 \\
\hline \multicolumn{4}{|l|}{ Medication before MI", No. (\%) } \\
\hline$\beta$-blockers & $20(8)$ & $43(8)$ & 0.900 \\
\hline Statins & $55(22)$ & $65(12)$ & $<0.001$ \\
\hline ACE/ARB blockers & $50(21)$ & $90(17)$ & 0.200 \\
\hline Aspirin & $28(12)$ & $40(8)$ & 0.060 \\
\hline
\end{tabular}

IQR: interquartile range; unit $^{\ddagger}$ of alcohol $=12 \mathrm{~g}$ (1 drink); COPD: chronic obstructive pulmonary disease; Ml: myocardial infarction; ACE/ARB: angiotensin-convertingenzyme inhibitor/ angiotensin II receptor blocker

VF in the setting of first STEMI and found no associations in the GEVAMI case-control population. To support the idea behind the hypothesis of this study several cohort studies including our GEVAMI study [1] have shown an increased risk for VF in patients with $\mathrm{AF}$ [4]. AF at initial presentation in the context of acute MI has also been associated with an increased risk of in-hospital VF in the FAST-MI 2005 registry [3]. Furthermore, a large Dutch population-based case-control study (the ARREST study) found similar increases in the risk of out-of-hospital VF arrest associated with a preceding diagnosis of AF [2]. In this study, 1397 out-of-hospital VF cases were compared with 3474 age- and sex-matched controls from the general population (i.e., without cardiac arrest). AF was associated with a threefold increased risk of VF $(\mathrm{OR}=3.1,95 \% \mathrm{CI}$ 2.1-4.5). Lastly, in a Danish cohort AF has been reported to cause an increased risk of SCD following acute myocardial infarction [6].

Also several genetic studies have supported the idea behind the hypothesis of this study. The first GWAS performed on AF identified an association of SNP rs2200733 on chromosome 4q25 with AF in those of European and Asian descent [27]. Since this important finding, several studies have found the same association 
Table 2 Additive genetic model of inheritance (per copy allele frequency) for association of 24 SNPs previously associated with atrial fibrillation, and in this study investigated for association with ventricular fibrillation before ST-segment elevation myocardial infarction

\begin{tabular}{|c|c|c|c|c|c|c|c|c|}
\hline & Locus & SNP & RA & RAF (Cases/Controls) & OR & $95 \% \mathrm{Cl}$ & $P$ & $\begin{array}{l}\text { Nearest gene } \\
\text { symbol }\end{array}$ \\
\hline $1 \&$ & $1 q 21$ & rs6666258 & $C$ & $0.31 / 0.33$ & 0.91 & $0.72-1.15$ & 0.400 & $\operatorname{KCNN3}[9,26]$ \\
\hline 2 & $1 \mathrm{q} 24$ & rs3903239 & $C$ & $0.46 / 0.49$ & 0.85 & $0.68-1.07$ & 0.200 & PRRX1 [9] \\
\hline 3 & $3 p 25$ & rs4642101 & G & $0.61 / 0.62$ & 0.99 & $0.79-1.25$ & 0.900 & CAND2 [22] \\
\hline $4 \#^{*}$ & $3 p 21$ & rs6795970 & A & $0.00 / 0.00$ & - & - & - & SCN1OA [17] \\
\hline $5 \&^{*}$ & $4 q 25$ & rs2200733 & T & $0.08 / 0.08$ & 0.96 & $0.65-1.42$ & 0.900 & PITX2 $[9,10]$ \\
\hline $6 \&$ & $4 q 25$ & rs2634073 & A & $0.14 / 0.15$ & 0.88 & $0.65-1.20$ & 0.400 & PITX2 [9] \\
\hline $7 \&$ & $4 q 25$ & rs6843082 & G & $0.16 / 0.18$ & 0.87 & $0.66-1.17$ & 0.400 & PITX2 $[9,14]$ \\
\hline 8 & $4 q 25$ & rs1448818 & G & $0.26 / 0.24$ & 1.13 & $0.89-1.43$ & 0.300 & PITX2 [21] \\
\hline $9 \&$ & $4 q 25$ & rs10033464 & T & $0.76 / 0.94$ & 0.79 & $0.53-1.17$ & 0.300 & PITX2 [21, 27] \\
\hline 10 & $4 q 25$ & rs17570669 & T & $0.06 / 0.08$ & 0.74 & $0.48-1.12$ & 0.200 & PITX2 [21] \\
\hline 11 & $4 q 25$ & rs2723288 & T & $0.29 / 0.28$ & 1.08 & $0.86-1.35$ & 0.500 & PITX2 [21] \\
\hline 12 & $4 q 25$ & rs4400058 & A & 0.08/0.09 & 0.80 & $0.54-1.18$ & 0.300 & PITX2 [21] \\
\hline 13 & $4 q 25$ & rs6838973 & T & $0.43 / 0.44$ & 0.95 & $0.76-1.18$ & 0.700 & PITX2 [21] \\
\hline 14 & $4 q 25$ & rs3853445 & $C$ & $0.26 / 0.25$ & 0.99 & $0.78-1.27$ & 0.900 & PITX2 [21] \\
\hline 15 & $5 q 31$ & rs2040862 & T & $0.18 / 0.16$ & 1.15 & $0.87-1.53$ & 0.300 & PITX2 [21] \\
\hline 16 & $6 q 22$ & rs13216675 & T & $0.68 / 0.69$ & 0.95 & $0.75-1.18$ & 0.600 & GJA1 [22] \\
\hline 17 & $7 q 31$ & rs3807989 & G & $0.41 / 0.42$ & 1.00 & $0.81-1.25$ & 0.900 & CAV1 [9] \\
\hline 18 & $9 q 22$ & rs10821415 & A & 0.39/0.39 & 0.97 & $0.78-1.20$ & 0.800 & C9ORF3 [9] \\
\hline 19 & $10 q 22$ & rs 10824026 & G & $0.13 / 0.15$ & 0.85 & $0.62-1.16$ & 0.300 & MYOZ1 [9] \\
\hline 20 & $10 q 24$ & rs 12415501 & T & $0.15 / 0.16$ & 0.92 & $0.68-1.23$ & 0.600 & NEURL [22] \\
\hline 21 & $12 q 24$ & rs 10507248 & T & $0.75 / 0.74$ & 1.05 & $0.82-1.33$ & 0.700 & TBX5 [22] \\
\hline 22 & $14 q 23$ & rs1152591 & T & $0.51 / 0.48$ & 1.11 & 0.89-1.38 & 0.300 & SYNE2 [9] \\
\hline 23 & $15 q 24$ & rs7164883 & G & $0.17 / 0.13$ & 1.28 & $0.96-1.70$ & 0.090 & HCN4 [9] \\
\hline 24 & $16 q 22$ & rs2106261 & A & $0.18 / 0.17$ & 1.09 & $0.83-1.44$ & 0.500 & ZFHX3 $[9,36]$ \\
\hline
\end{tabular}

SNP: single-nucleotide polymorphism; RA: Risk allele; RAF: Risk allele frequency in cases over controls in our cohort; OR: odds ratio; Cl: confidence interval; P value for the additive genetic model of inheritance (per copy allele frequency)

\#: No risk allele (A) exists in the GEVAMI cohort. Logistic regression models under an additive model of inheritance adjusted for age and sex. Number of cases $=257$; number of controls $=537$

\&: The SNP rs6666258 is in linkage disequilibrium $\left(r^{2}=1.00\right)$ with $r s 13376333$

\&: The SNP 2200733 is in linkage disequilibrium $\left(r^{2}=1.00\right)$ with $r 56817105$ and $r s 17042171$

\&: The rs2634073 is in linkage disequilibrium $\left(r^{2}=0.80\right)$ with $r 6843082$

\&: The rs10033464 is in linkage disequilibrium $\left(r^{2}=1.00\right)$ with $r s 4032974$

*: The SNP rs6795970 and 2,200,733 are both associated with SCD [20]

in other populations and also with an increased risk for prolonged PR interval, [28] which has been shown to be associated with all-cause mortality [29]. In a metaanalysis involving 716 SCD cases, the rs2200733 variant in 4q25 was found to be significantly associated with SCD (relative risk (RR) of 1.28 per minor T allele (95\% CI: $1.11-1.48 ; P=7.9 \times 10^{-4}$ ) [15]. The latter study supports a joint genetic pathway between AF and VF or at least abnormal cardiac function. The rs2200733 has been associated with expression of PITX2, which is a transcription factor, and the expression level of PITX2 has been associated with structural changes as well as expression of ion channel genes such as SCN5A [30].
Furthermore, the SNP rs6795970 which is in high linkage disequilibrium $\left(r^{2}=0.93\right)$ with $r s 6801957$ at the SCN10A locus has previously been associated with abnormality of cardiac conduction [16, 17, 23]. The SNP rs6795970 has also been associated with AF in two different studies [23, 24]. Furthermore, Chambers et al. identified that rs6795970 is associated with prolonged cardiac conduction (longer P-wave duration, PR interval and QRS duration) [17]. In contrast, in the same study [17] the rs6795970 was significantly protective against risk of VF caused by first STEMI among the 976 participants in the Arrhythmia Genetics in The NEtherlands (AGNES) study with an OR of 0.79 (95\% CI: 0.66-0.95; 
$p=0.01)$. In the GEVAMI cohort we were not able to show an association between the SNP rs6795970 and VF. Obviously, since the A risk allele did not exist in the current GEVAMI population. This could be due to the selected STEMI population, and according to our knowledge the frequency of this SNP is not reported in a general Danish population. However, as a proxy for the SNP rs6795970, we previously published another SNP (rs10428132) which is also located near the SCN10A gene at chromosome 3, and which is in highly linkage with SNP rs6795970 $\left(r^{2}=0.966\right)$ and found no association with VF [20]. However, the mechanisms linking genetic variants in SCN1OA with cardiac conduction remains to be determined. As mentioned earlier several studies have found rare and comment variants associated with AF. However the present AF-data only account for a very limited percentage of the heritability of $\mathrm{AF}$ and this is also the case for SCD and VF caused by STEMI. Therefore, identification of additional AF and VF loci by new technologies such as next generation sequencing may improve our knowledge of heritability of $\mathrm{AF}$ and VF and thereby understanding of if a shared genetic mechanism exists between the two arrhythmias.

There is also evidence for the proarrhythmic feature of AF, which by itself can induce VF without genetic involvment [31]. One study suggested that rapid ventricular rate during $\mathrm{AF}$ will reduce the ventricular refractoriness and induce ventricular tachyarrhythmia [32]. Moreover, the irregular rhythm of AF may cause VF through abrupt short-to-long changes in the cycle length, [33] and in AF patients with an implantable cardioverter defibrillator have more frequently and significantly induced VT/VF [34]. Lastly, AF by hemodynamic changes, can decrease parasympathetic tone and increase sympathetic tone making emerging of VF more likely [35].

The strength of the GEVAMI study is the prospective design and the use of a well-defined phenotype (VF). It is possible that some of these SNPs would have reached significance in a larger sample. In current study, the absolute number of patients with AF was low. A total of 16 (7\%) cases and 10 (2\%) controls were known with previous AF. Given our relatively low sample size, we acknowledge that this study may has been underpowered to detect associations. Most AF SNPs are high-frequency variants with small incremental effects on risk and therefore may limit the ability of this study to find association of AF-variants with small effect size. It is also important to mention that collecting cases is very time consuming and limited due to relatively low incidence of VF and difficulties to enroll and collect blood samples in STEMI-patients with VF and cardiac arrest. This is mostly due to that patients who died outside of the hospital or died in-hospital prior to enrollment cannot be included. The association of AF and VF in the cohort studies may be due to that AF patients have more comorbidity and therefore develop VF during acute ischemia. We only included patients with VF within $12 \mathrm{~h}$ before PPCI, therefore our data only represent patients with VF within this time interval since patients with late VF (VF after $12 \mathrm{~h}$, or VF during PPCI) were excluded. Finally, our results in this population with white, European ancestry may not be generalizable to other populations.

\section{Conclusion}

In this study we found that the 24AF-associated SNPs may not be involved in increasing the risk for VF. The complexity and interconnections between the two arrhythmias needs to be investigated in larger aspects and not only based on SNPs. Larger VF cohorts and use of new next generation sequencing and epigenetic may in future identify additional AF and VF risk loci and improve our understanding of genetic pathways behind the two arrhythmias.

\section{Abbreviations \\ AF: Atrial fibrillatiom; GEVAMI: GEnetic causes of Ventricular Arrhythmias in patients with first ST-elevation Myocardial Infarction; GWAS: Genome-wide association studies; IQR: Interquartile range; OR: Odds ratio; PPCl: Primary percutaneous coronary intervention; SCD: Sudden cardiac death; SNP: Single nucleotide polymorphism; STEMI: ST-segment elevation myocardial infarction; VF: Ventricular fibrillation}

\section{Acknowledgements}

This study was supported by research grants from the Novo Nordisk, The Research Foundation of the Heart Centre at Rigshospitalet, and The Research Foundation of the University Hospital of Copenhagen, Rigshospitalet. We acknowledge the support from different institutions.

\section{Funding}

This study was supported by research grants from the Novo Nordisk to Dr. Tfelt-Hansen, The Research Foundation of the Heart Centre at Rigshospitalet, and The Research Foundation of the University Hospital of Copenhagen, Rigshospitalet. The funders had no role in study design, data collection and analysis, decision to publish, or preparation of the manuscript. Dr. Javad Jabbari is an employee of LEO Pharma A/S, Denmark, but he was not employed by LEO Pharma A/S during the course of the study. The ESCAPENET project. The ESCAPE-NET project has received funding from the European Union's Horizon 2020 research and innovation programme under grant agreement No 733381

\section{Availability of data and materials}

The datasets used and/or analysed during the current study available from the corresponding author on reasonable request.

\section{Disclosures}

Dr. Javad Jabbari is an employee of LEO Pharma A/S, Denmark.

\section{Authors' contributions}

RJ conceived and designed the study, analyzed the data, and wrote the paper; JJ, CG, BR, SS, and BGW helped analyzing the data, and writing the paper; CJT, HHT, LOJ, MH, and $\mathrm{SH}$, designed the study and reviewed the paper. TE, CA, and JTH designed, organized and supervised the project. All authors read and approved the final manuscript.

\section{Ethics approval and consent to participate}

Signed informed consent is available for all patients enrolled in this study. Procedures are in accordance with the ethical standards of the National Committee (the Capital Region of Denmark) on Health Research Ethics (protocol number: H-3-2010-133) and with the Helsinki Declaration of 1975, 
as revised in 1983. Permission from the Danish Data Protection Agency was also obtained before the study was initiated (Jr.nr. 2010-41-5688). The study was conducted according to the guidelines of The National Committee on Health Research Ethics, Denmark.

\section{Consent for publication}

\section{Not applicable.}

\section{Competing interests}

The authors declare they have no competing interests.

\section{Publisher's Note}

Springer Nature remains neutral with regard to jurisdictional claims in published maps and institutional affiliations.

\section{Author details}

${ }^{1}$ Department of Cardiology, Rigshospitalet, Copenhagen University Hospital, Blegdamsvej 9, 2100 Copenhagen $\varnothing$, Denmark. ${ }^{2}$ Department of Cardiology, Aarhus University Hospital, Skejby, Nørrebrogade, 44, 8000 Aarhus C, Denmark. ${ }^{3}$ Department of Cardiology, Aalborg University Hospital, Hobrovej 18-22, 9100 Aalborg, Denmark. ${ }^{4}$ Department of Cardiology, Odense University Hospital, Søndre Blvd. 29, 5000 Odense C, Denmark. ${ }^{5}$ Laboratory of Molecular Cardiology, Department of Cardiology, Copenhagen University Hospital Rigshospitalet, Juliane Mariesvej 20, 2100 Copenhagen $\varnothing$, Denmark. ${ }^{6}$ Center for Arrhythmia Prevention, Division of Preventive Medicine, Cardiovascular Division, Department of Medicine, Brigham and Women's Hospital, Harvard Medical School, 75 Francis Street, Boston, MA 02115, USA. ${ }^{7}$ Department of Cardiology, University of Lund, Lund, Sweden.

\section{Received: 1 March 2017 Accepted: 9 November 2017}

\section{Published online: 21 November 2017}

\section{References}

1. Jabbari R, Engstrøm T, Glinge C, Risgaard B, Jabbari J, Winkel BG, et al. Incidence and risk factors of ventricular fibrillation before primary angioplasty in patients with first ST-elevation myocardial infarction: a nationwide study in Denmark. J Am Heart Assoc. 2015 Jan;4(1):e001399.

2. Bardai A, Blom MT, van Hoeijen DA, van Deutekom HW, Brouwer HJ, Tan $\mathrm{HL}$. Atrial Fibrillation Is An independent risk factor for ventricular fibrillation: a large-scale population-based case-control study. Circ Arrhythm Electrophysiol 2014 Sep 18;

3. Bougouin W, Marijon E, Puymirat E, Defaye P, Celermajer DS, Le Heuzey J-Y, et al. Incidence of sudden cardiac death after ventricular fibrillation complicating acute myocardial infarction: a 5-year cause-of-death analysis of the FAST-MI 2005 registry. Eur Heart J. 2014 Jan;35(2):116-22.

4. Jabbari R. Ventricular fibrillation and sudden cardiac death during myocardial infarction. Dan Med J. 2016;63(5). Review.

5. Chen LY, Sotoodehnia N, Bůžková P, Lopez FL, Yee LM, Heckbert SR, et al. Atrial fibrillation and the risk of sudden cardiac death: the atherosclerosis risk in communities study and cardiovascular health study. JAMA Intern Med. 2013 Jan 14;173(1):29-35

6. Pedersen OD, Abildstrøm SZ, Ottesen MM, Rask-Madsen C, Bagger H, Køber $L$, et al. Increased risk of sudden and non-sudden cardiovascular death in patients with atrial fibrillation/flutter following acute myocardial infarction. Eur Heart J. 2006 Feb;27(3):290-5.

7. Reinier K, Marijon E, Uy-Evanado A, Teodorescu C, Narayanan K, Chugh H, et al. The association between atrial fibrillation and sudden cardiac death: the relevance of heart failure. JACC Heart Fail. 2014 Jun;2(3):221-7.

8. Marsman RF, Tan HL, Bezzina CR. Genetics of sudden cardiac death caused by ventricular arrhythmias. Nat Rev Cardiol. 2014 Feb;11(2):96-111.

9. Tucker NR, Ellinor PT. Emerging directions in the genetics of atrial fibrillation. Circ Res. 2014 Apr 25;114(9):1469-82.

10. Kääb S, Darbar D, van Noord C, Dupuis J, Pfeufer A, Newton-Cheh C, et al. Large scale replication and meta-analysis of variants on chromosome $4 \mathrm{q} 25$ associated with atrial fibrillation. Eur Heart J. 2009 Apr;30(7):813-9.

11. Viviani Anselmi C, Novelli V, Roncarati R, Malovini A, Bellazzi R, Bronzini R, et al. Association of rs 2200733 at 4q25 with atrial flutter/fibrillation diseases in an Italian population. Heart Br Card Soc. 2008 Nov;94(11):1394-6.

12. Shi L, Li C, Wang C, Xia Y, Wu G, Wang F, et al. Assessment of association of rs2200733 on chromosome 4q25 with atrial fibrillation and ischemic stroke in a Chinese Han population. Hum Genet. 2009 Dec;126(6):843-9.
13. Delaney JT, Jeff JM, Brown NJ, Pretorius M, Okafor HE, Darbar D, et al. Characterization of genome-wide association-identified variants for atrial fibrillation in African Americans. PLoS One. 2012;7(2):e32338.

14. Ellinor PT, Lunetta KL, Albert CM, Glazer NL, Ritchie MD, Smith AV, et al. Meta-analysis identifies six new susceptibility loci for atrial fibrillation. Nat Genet. 2012 Jun;44(6):670-5.

15. Lahtinen AM, Noseworthy PA, Havulinna AS, Jula A, Karhunen PJ, Kettunen J, et al. Common genetic variants associated with sudden cardiac death: the FinSCDgen study. PLoS One. 2012;7(7):e41675.

16. Sotoodehnia N, Isaacs A, de Bakker PIW, Dörr M, Newton-Cheh C, Nolte IM, et al. Common variants in 22 loci are associated with QRS duration and cardiac ventricular conduction. Nat Genet. 2010 Dec;42(12):1068-76.

17. Chambers JC, Zhao J, Terracciano CMN, Bezzina CR, Zhang W, Kaba R, et al. Genetic variation in SCN10A influences cardiac conduction. Nat Genet. 2010 Feb;42(2):149-52.

18. Behr ER, Savio-Galimberti E, Barc J, Holst AG, Petropoulou E, Prins BP, et al. Role of common and rare variants in SCN10A: results from the Brugada syndrome QRS locus gene discovery collaborative study. Cardiovasc Res. 2015 Jun 1;106(3):520-9.

19. Tfelt-Hansen J, Winkel BG, Grunnet M, Jespersen T. Inherited cardiac diseases caused by mutations in the Nav1.5 sodium channel. J Cardiovasc Electrophysiol. 2010 Jan;21(1):107-15.

20. Jabbari R, Glinge C, Jabbari J, Risgaard B, Winkel BG, Terkelsen CJ, et al. A common variant in SCN5A and the risk of ventricular fibrillation caused by first ST-segment elevation myocardial infarction. PLoS One. 2017;12(1):e0170193.

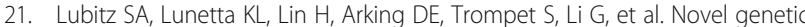
markers associate with atrial fibrillation risk in Europeans and Japanese. J Am Coll Cardiol. 2014 Apr 1;63(12):1200-10.

22. Sinner MF, Tucker NR, Lunetta KL, Ozaki K, Smith JG, Trompet S, et al. Integrating genetic, transcriptional, and functional analyses to identify 5 novel genes for atrial fibrillation. Circulation. 2014 Oct 7;130(15):1225-35.

23. Pfeufer A, van Noord C, Marciante KD, Arking DE, Larson MG, Smith AV, et al. Genome-wide association study of PR interval. Nat Genet. 2010 Feb;42(2):153-9.

24. Ritchie MD, Denny JC, Zuvich RL, Crawford DC, Schildcrout JS, Bastarache L, et al. Genome- and phenome-wide analyses of cardiac conduction identifies markers of arrhythmia risk. Circulation. 2013 Apr 2;127(13):1377-85.

25. Olesen MS, Nielsen MW, Haunsø S, Svendsen JH. Atrial fibrillation: the role of common and rare genetic variants. Eur J Hum Genet EJHG. 2014 Mar; 22(3):297-306.

26. Ellinor PT, Lunetta KL, Glazer NL, Pfeufer A, Alonso A, Chung MK, et al. Common variants in KCNN3 are associated with lone atrial fibrillation. Nat Genet. 2010 Mar:42(3):240-4.

27. Gudbjartsson DF, Arnar DO, Helgadottir A, Gretarsdottir S, Holm H, Sigurdsson A, et al. Variants conferring risk of atrial fibrillation on chromosome 4q25. Nature. 2007 Jul 19;448(7151):353-7.

28. Kolek MJ, Parvez B, Muhammad R, Shoemaker MB, Blair MA, Stubblefield T, et al. A common variant on chromosome $4 \mathrm{q} 25$ is associated with prolonged PR interval in subjects with and without atrial fibrillation. Am J Cardiol. 2014 Jan 15;113(2):309-13.

29. Cheng S, Keyes MJ, Larson MG, McCabe EL, Newton-Cheh C, Levy D, et al. Long-term outcomes in individuals with prolonged PR interval or firstdegree atrioventricular block. JAMA. 2009 Jun 24;301(24):2571-7.

30. Chinchilla A, Daimi H, Lozano-Velasco E, Dominguez JN, Caballero R, Delpón E, et al. PITX2 insufficiency leads to atrial electrical and structural remodeling linked to arrhythmogenesis. Circ Cardiovasc Genet. 2011 Jun;4(3):269-79.

31. Chen LY, Benditt DG, Alonso A. Atrial fibrillation and its association with sudden cardiac death. Circ J Off J Jpn Circ Soc. 2014:78(11):2588-93.

32. Denes $P$, Wu D, Dhingra R, Pietras RJ, Rosen KM. The effects of cycle length on cardiac refractory periods in man. Circulation. 1974 Jan;49(1):32-41.

33. Denker S, Lehmann M, Mahmud R, Gilbert C, Akhtar M. Facilitation of ventricular tachycardia induction with abrupt changes in ventricular cycle length. Am J Cardiol. 1984 Feb 1;53(4):508-15.

34. Grönefeld GC, Mauss O, Li YG, Klingenheben T, Hohnloser SH. Association between atrial fibrillation and appropriate implantable cardioverter defibrillator therapy: results from a prospective study. J Cardiovasc Electrophysiol. 2000 Nov; 11(11):1208-14.

35. Shen MJ, Zipes DP. Role of the autonomic nervous system in modulating cardiac arrhythmias. Circ Res. 2014 Mar 14;114(6):1004-21.

36. Benjamin EJ, Rice KM, Arking DE, Pfeufer A, van Noord C, Smith AV, et al. Variants in ZFHX3 are associated with atrial fibrillation in individuals of European ancestry. Nat Genet. 2009 Aug;41(8):879-81. 